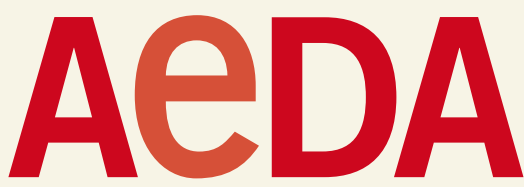

Ärzteverband Deutscher Allergologen (AeDA)

\section{Verantwortlich für den Inhalt}

Prof. Dr. Ludger Klimek Zentrum für Rhinologie und Allergologie An den Quellen 10, 65183 Wiesbaden Tel.: (06 11) 8804799 Fax: (06 11) 308608255 E-Mail: ludger.klimek@allergiezentrum.org

\section{AeDA-Geschäftsstelle}

Service Systems

Blumenstraße 14, 63303 Dreieich

Tel.: (0 61 03) 62273

Fax: (0 61 03) 697019

E-Mail: info@aeda.de

www.aeda.de

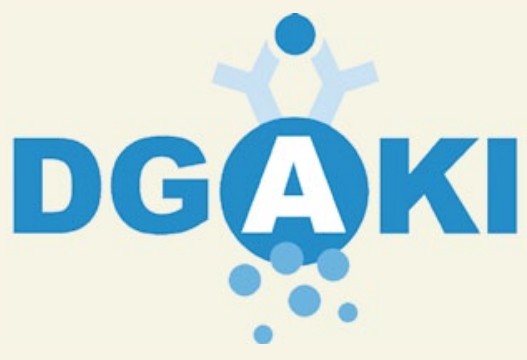

Deutsche Gesellschaft für Allergologie und klinische Immunologie (DGAKI)

\section{Verantwortlich für die Inhalte}

Prof. Dr. Thilo Jakob

Allergieabteilung und Forschergruppe Allergologie, Universitäts-Hautklinik Freiburg Hauptstraße 7, 79104 Freiburg

Tel.: (07 61) 2706714

Fax: (07 61) 2706655

E-Mail: thilo.jakob@uniklinik-freiburg.de

\section{DGAKI-Geschäftsstelle}

Gertrud Hammel

Schloss 2, 86482 Aystetten

Tel.: (08 21) 48687864

Fax: (08 21) 48687863

E-Mail: info@dgaki.de

www.dgaki.de

\section{Forschungsatlas Pädiatrische Allergologie erschienen}

Wer forscht in Deutschland im Bereich der pädiatrischen Allergologie? Diese Frage beantwortet der neue „Forschungsatlas Pädiatrische Allergologie“. Es gibt über 30 Zentren, die in diesem Bereich tätig sind, erklärt Co-Herausgeber PD Dr. Christian Vogelberg, Dresden: „Um diese Wissenschaftler besser miteinander zu vernetzen und ihre Arbeiten bekannt zu machen, hat die Wissenschaftliche Arbeitsgruppe Forschung der Gesellschaft für

Pädiatrische Allergologie und Umweltmedizin (GPA)

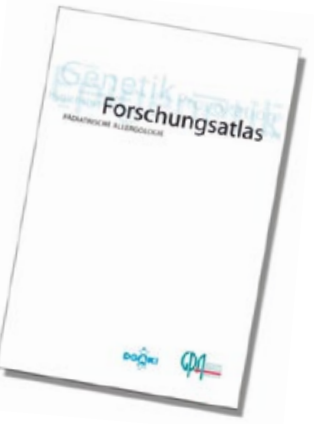

zusammen

mit der Sektion Pädiatrie

der DGAKI diesen Forschungsatlas herausgegeben. “Der Atlas kann über die GPAGeschäftsstelle (gpa.ev@t-online.de) bestellt werden.

\title{
Ausgezeichnete Asthmaforscherinnen
}

Dr. Anne M. Fitzpatrick, Assistant Professor an der Emory University School of Medicine in Atlanta, USA, versucht, schweres Asthma bei Kindern klinisch und molekularbiologisch genauer zu charakterisieren. Sie fand eine hohe Heterogenität der klinischen Phänotypen und konnte einen besonderen molekularen Phänotyp identifizieren. Zudem zeigte sie, wie antioxidative Schutzmechanismen bei schwerem Asthma außer Kraft gesetzt werden. Ihre Erkenntnisse um die verschiedenen Endotypen könnten langfristig zu einer verbesserten Therapie führen und waren für die Jury des „International Klosterfrau Award Research of Airway Diseases in Childhood 2012" mehr als Grund genug, Fitzpatrick auszuzeichnen.

Der zweite Preis, vergeben als „Research Grant", ging an Dr. Anna-Maria Dittrich vom Zentrum Kinderheilkunde und Jugendmedizin der Medizinischen Hochschule Hannover. Ihr gelang der Nachweis, dass auch eine Entzündung der Atemwege, die allein durch TH17-polarisierte T-Helferzellen induziert wird, die Sensibilisierung auf neue Antigene erleichtert.

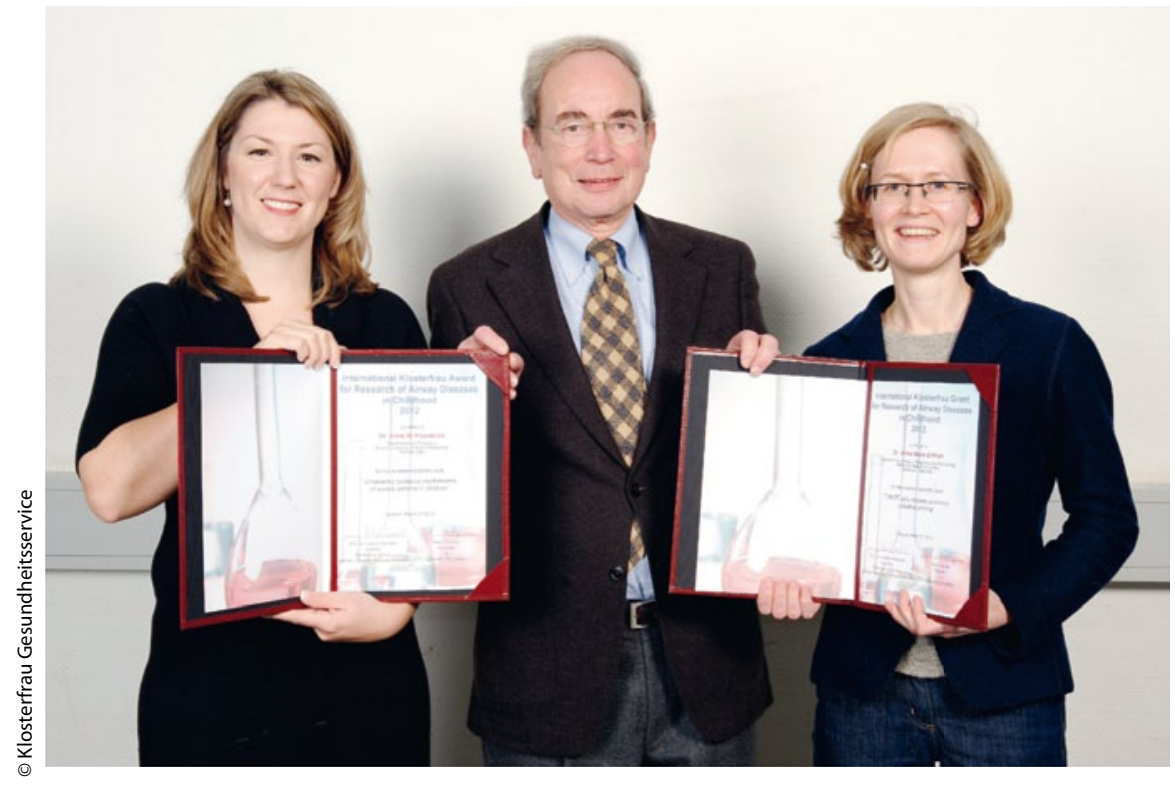

Die Klosterfrau-Preisträgerinnen Dr. Anne M. Fitzpatrick (links) und Dr. Anna-Maria Dittrich rahmen Jurypräsident Prof. Dr. Dietrich Reinhardt ein. 